\title{
SEVEN-DIMENSIONAL CONSIDERATIONS OF EINSTEIN'S CONNECTION. II. THE RECURRENCE RELATIONS OF THE SECOND AND THIRD KIND IN 7 - $g$-UFT
}

\author{
MI AE KIM, KEUM SOOK SO, CHUNG HYUN CHO, \\ and KYUNG TAE CHUNG
}

Received 12 April 2001 and in revised form 27 September 2001

\begin{abstract}
We present powerful recurrence relations of the second and third kind which hold in 7-dimensional Einstein's generalized Riemannian manifold $X_{7}$. All considerations in this paper are restricted to the first and second classes of $X_{7}$ only since the case of the third class, the simplest case, was already studied by many authors.
\end{abstract}

2000 Mathematics Subject Classification: 83E50, 83C05, 58A05.

Einstein's connection in a generalized Riemannian manifold $X_{n}$ has been investigated by many authors for lower-dimensional cases $n=2, \ldots, 6$. In a series of papers, we obtain a surveyable tensorial representation of 7-dimensional Einstein's connection in terms of unified field tensor, with main emphasis on the derivation of powerful and useful recurrence relations which hold in $X_{7}$.

In [1], which we denote by I in the present paper, we gave a brief survey of Einstein's unified field theory and derive the recurrence relations of the first kind which hold in a general $X_{n}$. We continue our investigations in this paper and derive a powerful recurrence relations of the second and third kinds which hold in 7-dimensional Einstein's generalized Riemannian manifold $X_{7}$. These relations will be used in a subsequent paper to find a tensorial representation of 7-dimensional Einstein's connection. All considerations in this paper are based on the results and symbolism of I. Whenever necessary, they will be quoted in the present paper.

In order to obtain a tensorial representation of the 7-dimensional Einstein's connection $\Gamma_{\lambda \mu}^{\nu}$ in terms of $g_{\lambda \mu}$, we need powerful recurrence relations of the third kind satisfied by an arbitrary tensor $T_{\omega \lambda \mu}$, skew-symmetric in the first two indices. Therefore, we derive these relations in this paper after introducing the recurrence relations of the second kind satisfied by the basic scalars. All considerations in this paper are restricted to $n=7$.

In 7 - $g$-UFT, there are four cases, that is, the unified field tensor $g_{\lambda \mu}$ belongs to

(1) the first class, if $K_{6} \neq 0$

(2) the second class with the first category, if $K_{2} \neq 0, K_{4}=K_{6}=0$ 
(3) the second class with the second category, if $K_{4} \neq 0, K_{6}=0$

(4) the third class, if $K_{2}=K_{4}=K_{6}=0$.

In this paper, we investigate the first three cases.

Before we start investigations about the basic scalars, we first note that in $[1$, relation (2.10)], $7-g$-UFT is reduced to

$$
g=1+K_{2}+K_{4}+K_{6}
$$

In the following theorem, we formally state the recurrence relations of the first kind when $n=7$, direct consequences of [1, (3.1) and (3.2)].

THEOREM 1 (the recurrence relations of the first kind in 7-g-UFT). The tensors ${ }^{(p)} k_{\lambda}{ }^{v}$ satisfy the following recurrence relations in $7-g$-UFT for $p=$ $0,1,2, \ldots$ :

(i) the first class

$$
{ }^{(p+7)} k_{\lambda}{ }^{v}=-K_{2}{ }^{(p+5)} k_{\lambda}{ }^{v}-K_{4}{ }^{(p+3)} k_{\lambda}{ }^{v}-K_{6}{ }^{(p+1)} k_{\lambda}{ }^{v},
$$

(ii) the second class with the second category

$$
{ }^{(p+5)} k_{\lambda}{ }^{v}=-K_{2}^{(p+3)} k_{\lambda}^{v}-K_{4}^{(p+1)} k_{\lambda}^{v},
$$

(iii) the second class with the first category

$$
{ }^{(p+3)} k_{\lambda} v=-K_{2}{ }^{(p+1)} k_{\lambda}{ }^{v} \text {. }
$$

THEOREM 2. The basic scalars in 7-g-UFT are given by

(i) the first class

$$
\begin{aligned}
& \underset{1}{M}=-\underset{2}{M}=\sqrt{-\frac{K_{2}}{3}+\alpha+\beta}, \\
& \underset{3}{M}=-\underset{4}{M}=\sqrt{-\frac{K_{2}}{3}+\omega \alpha+\omega^{2} \beta}, \\
& \underset{5}{M}=-\underset{6}{M}=\sqrt{-\frac{K_{2}}{3}+\omega^{2} \alpha+\omega \beta}, \\
& \underset{7}{M}=0,
\end{aligned}
$$

(ii) the second class with the second category

$$
\begin{aligned}
& \underset{1}{M}=\underset{2}{M}=\sqrt{-L-K}, \\
& \underset{3}{M}=\underset{4}{M}=\sqrt{L-K}, \\
& \underset{5}{M}=\underset{6}{M}=\underset{7}{M}=0,
\end{aligned}
$$


(iii) the second class with the first category

$$
\begin{aligned}
& \underset{1}{M}=\underset{2}{-}=\sqrt{-K_{2}} \neq 0, \\
& \underset{3}{M}=\underset{4}{M}=\underset{5}{M}=\underset{6}{M}=\underset{7}{M}=0,
\end{aligned}
$$

where

$$
\begin{aligned}
& \omega=\frac{-1+\sqrt{3} i}{2}, \\
& \alpha=\left[-\frac{\phi}{2}+\sqrt{\left(\frac{\phi}{2}\right)^{2}+\left(\frac{\theta}{3}\right)^{3}}\right]^{1 / 3}, \\
& \beta=\left[-\frac{\phi}{2}-\sqrt{\left(\frac{\phi}{2}\right)^{2}+\left(\frac{\theta}{3}\right)^{3}}\right]^{1 / 3}, \\
& \theta=K_{4}-\frac{\left(K_{2}\right)^{2}}{3}, \\
& \phi=K_{6}-\frac{K_{2} K_{4}}{3}+\frac{2}{27}\left(K_{2}\right)^{3}, \\
& L=\sqrt{\left(\frac{K_{2}}{2}\right)^{2}-K_{4}}, \quad K=\frac{K_{2}}{2} .
\end{aligned}
$$

Proof. Since the characteristic equation [1, (2.15)] for the first class in 7 $g$-UFT is reduced to

$$
M\left(M^{2}\right)^{3}+K_{2}\left(M^{2}\right)^{2}+K_{4} M^{2}+K_{6}=0
$$

(3a) follows by the method of Cardano, using the notations given by (4). The other cases may be proved similarly.

THEOREM 3. The basic scalars $\underset{x}{M}$ in $7-g$-UFT satisfy the following relations:

(i) the first class

$$
\begin{aligned}
& \underset{1}{M}+\underset{2}{M}=\underset{3}{M}+\underset{4}{M}=\underset{5}{M}+\underset{6}{M}=0 \text {, } \\
& \underset{a}{M^{2}}+\underset{b}{M^{2}}+\underset{c}{M^{2}}=-K_{2}, \\
& \underset{a}{M_{b}^{2}} \underset{b}{M^{2}}+\underset{b}{M^{2}} \underset{c}{M^{2}}+\underset{c}{M^{2}} \underset{a}{M^{2}}=K_{4}, \\
& \underset{a}{M_{b}^{2}} \underset{c}{M^{2}} \underset{c}{M^{2}}=-K_{6}, \\
& \underset{a d}{M} \underset{b}{M}=\underset{c}{M}=\underset{d}{M} M=0,
\end{aligned}
$$


(ii) the second class with the second category

$$
\begin{aligned}
& \underset{1}{M}+\underset{2}{M}=\underset{3}{M}+\underset{4}{M}=\underset{5}{M}+\underset{6}{M}=0, \\
& \underset{a}{M} \underset{c}{M}=\underset{b}{M} \underset{c}{M}=\underset{a}{M} \underset{d}{M}=\underset{b d}{M M}=\underset{c}{M} \underset{d}{M}=0, \\
& \underset{a}{M^{2}}+\underset{b}{M^{2}}=-K_{2}, \\
& \underset{a}{M^{2}} \underset{b}{M^{2}}=K_{4}, \\
& \underset{a}{M_{c}^{2}} \underset{c}{M^{2}}=\underset{b}{M^{2}} \underset{c}{M^{2}}=0 \text {, }
\end{aligned}
$$

(iii) the second class with the first category

$$
\begin{gathered}
\underset{1}{M}+\underset{2}{M}=\underset{x}{M}+\underset{y}{M}=0, \\
\underset{12}{M M_{1}}=K_{2}, \quad \underset{1}{M} \underset{x}{M}=\underset{2}{M} \underset{x}{M}=\underset{x}{M} M_{y}^{M}=0 .
\end{gathered}
$$

Here, the indices $a, b, c$, and $d$ are assumed to take values as $a=1,2, b=3,4$, $c=5,6$, and $d=7$.

Proof. Relations (11), (12), and (13) follow from (3a), (3b), and (3c), respectively. In the proof of (6), the following relations are used:

$$
\begin{gathered}
\omega^{2}=-1-\omega, \quad \omega^{3}=1, \\
\alpha \beta=-\frac{1}{3} K_{4}+\frac{1}{9}\left(K_{2}\right)^{2}, \quad \alpha^{3}+\beta^{3}=-K_{6}+\frac{K_{2} K_{4}}{3}-\frac{2}{27}\left(K_{2}\right)^{3} .
\end{gathered}
$$

Using the relations given in Theorem 3, we may prove the recurrence relations of the second kind in the following theorem.

THEOREM 4 (the recurrence relations of the second kind in 7-g-UFT). In 7 $g$-UFT, the basic scalars $\underset{x}{M}$ satisfy the following recurrence relations which hold for all values of $x$ and $y$ when $x \neq y$.

For the first class,

$$
\begin{aligned}
& \underset{x}{M^{(6}} \underset{y}{M^{1)}}=-\underset{x}{M^{(5}} \underset{y}{M^{2)}}-\underset{x}{M^{(4}} \underset{y}{M^{3)}}-K_{2} \underset{x}{M^{(4}} \underset{y}{M^{1)}}-K_{2} \underset{x}{M^{(3}} \underset{y}{M^{2)}}-K_{4} \underset{x}{M^{(2}} \underset{y}{M^{1)}}, \\
& \underset{x}{2} \underset{y}{(6}{\underset{y}{M}}^{2)}=-2 \underset{x}{M^{(5}} \underset{y}{M^{3)}}-\underset{x}{M^{4}} \underset{y}{M^{4}}-2 \underset{x}{K_{2}} \underset{x}{M^{(4}} \underset{y}{M^{2)}}-K_{2} \underset{x}{M^{3}} \underset{y}{M^{3}}-K_{4} \underset{x}{M^{2}} \underset{y}{M^{2}}+K_{6} \underset{x}{M} \underset{y}{M}, \\
& \underset{x}{M^{(6}} \underset{y}{M^{3)}}=-\underset{x}{M^{(5}} \underset{y}{M^{4)}}-K_{2} \underset{x}{M^{(4}} \underset{y}{M^{3)}}+K_{6} \underset{x}{M^{(2}} \underset{y}{M^{1)}},
\end{aligned}
$$

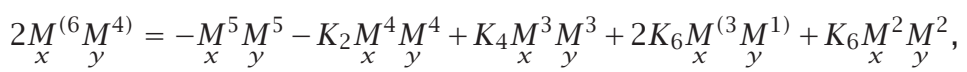

$$
\begin{aligned}
& \underset{x}{M^{(6}} \underset{y}{M^{5)}}=K_{4} \underset{x}{M^{(4}} \underset{y}{M^{3)}}+K_{6} \underset{x}{M^{(4}} \underset{y}{M^{1)}}+K_{6} \underset{x}{M^{(3}}{\underset{y}{M}}^{2)}, \\
& \underset{x}{M^{6}}{\underset{y}{6}}^{6}=K_{2} \underset{x}{M^{5}} \underset{y}{M^{5}}+2 K_{4} \underset{x}{M^{(5}}{ }_{y}^{M^{3)}}+2 K_{6} \underset{x}{M^{(5}} \underset{y}{M^{1)}}+K_{4} \underset{x}{M^{4}} \underset{y}{M^{4}}+2 K_{6} \underset{x}{M^{(4}} \underset{y}{M^{2)}}+K_{6} \underset{x}{M^{3}} \underset{y}{M^{3}} .
\end{aligned}
$$


For the second class with the second category,

$$
\begin{aligned}
& \underset{x}{M_{y}^{(4}} \underset{y}{M^{1)}}=-\underset{x}{M_{y}^{(3}} \underset{y}{M^{2)}}-K_{2} \underset{x}{M^{(2}} \underset{y}{M^{1)}}, \\
& \underset{x}{2} \underset{y}{(4}{ }_{y}^{2)}=-\underset{x}{M} \underset{y}{M^{3}}-K_{2} \underset{x}{M^{2}} \underset{y}{M^{2}}+K_{4} \underset{x}{M} \underset{y}{M}, \\
& \underset{x}{M^{(4}} \underset{y}{M^{3)}}=K_{4} \underset{x}{M^{(2}} \underset{y}{M^{1)}} \text {, } \\
& \underset{x}{M^{4}} \underset{y}{M^{4}}=K_{2} \underset{x}{M^{3}} \underset{y}{M^{3}}+2 K_{4} \underset{x}{M_{y}^{(3}} \underset{y}{M^{1)}}+K_{4} \underset{x}{M^{2}} \underset{y}{M^{2}} .
\end{aligned}
$$

For the second class with the first category,

$$
\underset{x}{M^{2}} \underset{y}{M^{2}}=K_{2} \underset{x}{M} \underset{y}{M}, \quad \underset{x}{M_{y}^{(2}} M^{1)}=0
$$

THE PROOF OF THE RELATIONS IN (15). The values of indices $x, y$ belong to any of the following four groups:

$$
x, y=a=1,2 ; \quad x, y=b=3,4 ; \quad x, y=c=5,6 ; \quad x, y=d=7 .
$$

For the values of two different indices $x$ and $y$, we have two cases. The first case is that $x$ and $y$ belong to the same group. The second case is that each of $x$ and $y$ belongs to two different groups. In the first case, it may be easily shown that the relations in (15) are identities $0=0$, because in the proof given below, each of $A, B, C, D, E, F$, and $G$ contains the factor $\underset{x}{M}+\underset{y}{M}=0$. Therefore, in the proof of relations (15), it suffices to show the validity of the relations for the second case only. Now let $x, y$, and $z$ be indices, each of which belongs to mutually different groups.

In order to prove (15a), consider

$$
A=\underset{x}{\operatorname{Ma}} \underset{y}{M}(\underset{y}{M}+\underset{x}{M})\left(\underset{y}{M^{2}} M^{2}+\underset{z}{M^{2}} \underset{z}{M^{2}}+\underset{z}{M^{2}} \underset{x}{M^{2}}\right)
$$

In virtue of (11c), we have

$$
A=2 K_{4} \underset{x}{M^{(2}} \underset{y}{M^{1)}}
$$

On the other hand, relation (11b) gives

$$
\begin{aligned}
& \left.A=\underset{x}{2 M_{y}^{(2}}{\underset{y}{1)}}^{1)} \underset{x}{M^{2}} \underset{y}{M^{2}}+\underset{y}{M^{2}}\left(-K_{2}-\underset{x}{M^{2}}-\underset{y}{M^{2}}\right)+\underset{x}{M^{2}}\left(-K_{2}-\underset{x}{M^{2}}-\underset{y}{M^{2}}\right)\right] \\
& =\underset{x}{2 M_{y}^{(2} M^{1)}}\left[-2 K_{2}\left(\underset{x}{M^{(2} M^{0)}}\right)-\underset{x}{2 M^{(4} M^{0)}}-\underset{x}{M^{2}} M^{2}\right] \\
& =-2 K_{2} \underset{x}{M^{(4}} \underset{y}{M^{1)}}-2 K_{2} \underset{x}{M^{(3}} \underset{y}{M^{2)}}-2 \underset{x}{M^{(6}} \underset{y}{M^{1)}}-2 \underset{x}{2}{ }^{(5} \underset{y}{M^{2)}}-\underset{x}{2 \underset{y}{(4}{ }_{y}^{3)}} \text {. }
\end{aligned}
$$

Consequently, relation (15a) follows from (20). 
On the other hand, for the proof of (15b), we consider

$$
B=(\underset{x}{M}+\underset{y}{M}) \underset{x}{M_{y}^{(6}} M^{1)}
$$

Then, relation (10) gives

$$
\begin{aligned}
& B=2 \underset{x}{M^{(1}} \underset{y}{M^{0)}} \underset{x}{M^{(6}} \underset{y}{M^{1)}}=\underset{x}{M^{(7}} \underset{y}{M^{1)}}+\underset{x}{M^{(6}} \underset{y}{M^{2)}} \\
& =-K_{2} \underset{x}{M^{(5}} \underset{y}{M^{1)}}-K_{4} \underset{x}{M^{(3}} \underset{y}{M^{1)}}-K_{6} \underset{x}{M} \underset{y}{M}+\underset{x}{M^{(6}} \underset{y}{M^{2)}} .
\end{aligned}
$$

Making use of (15a), we also have

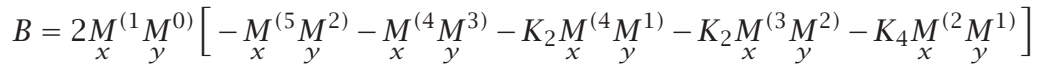

$$
\begin{aligned}
& =-\underset{x}{M^{(6}}{\underset{y}{M}}^{2)}-\underset{x}{M^{(5}}{\underset{y}{M}}^{3)}-K_{2} \underset{x}{M^{(5}} \underset{y}{M^{1)}}-2 K_{2} \underset{x}{M^{(4}}{ }_{y}^{M^{2)}} \\
& -K_{4} \underset{x}{M_{y}^{(3}} \underset{y}{M^{1)}}-\underset{x}{M^{4}} \underset{y}{M^{4}}-K_{2} \underset{x}{M^{3}} \underset{y}{M^{3}}-K_{4} \underset{x}{M^{2}} \underset{y}{M^{2}}
\end{aligned}
$$

Thus, comparing (22) with (23), we get (15b).

The remaining relations in (15) may be proved similarly by considering

$$
\begin{aligned}
& C=\underset{x}{M} \underset{y}{M}(\underset{x}{M}+\underset{y}{M}) \underset{x}{M^{2}} \underset{y}{M^{2}} \underset{z}{M^{2}} \text {, for the proof of (15c), } \\
& D=(\underset{x}{M}+\underset{y}{M}) \underset{x}{M^{(6}} \underset{y}{M^{3)}}, \quad \text { for the proof of (15d), } \\
& E=(\underset{x}{M} \underset{y}{M}) \underset{x}{M}{ }_{y}^{(6} M^{4)}, \quad \text { for the proof of (15e), } \\
& F=\underset{x}{M} \underset{y}{M}(\underset{x}{M}+\underset{y}{M}) \underset{x}{M^{(6} M^{3)}}, \quad \text { for the proof of (15f). }
\end{aligned}
$$

The PROOF OF THE ReLATIONS IN (16) AND (17). These relations may be obtained from (15) by substituting the corresponding conditions of each case.

Now, we are ready to prove the recurrence relations of the third kind in the following theorem. These relations are very important for the solution of [1, (2.5) or (2.26)] in 7-g-UFT. We use these relations in our subsequent paper to establish a linear system equivalent to $[1,(2.5)]$ and to find a precise and surveyable tensorial representation of 7-dimensional Einstein's connection in terms of the unified field tensor $g_{\lambda \mu}$. 
THEOREM 5 (the recurrence relations of the third kind in 7-g-UFT). If $T \equiv$ $T_{\omega \lambda \mu}$ is a skew-symmetric tensor in the first two indices, the following recurrence relations hold in $7-g-U F T$.

For the first class,

$$
\begin{aligned}
& \stackrel{(61) r}{T}=-\stackrel{(52) r}{T}-\stackrel{(43) r}{T}-K_{2} \stackrel{(41) r}{T}-K_{2} \stackrel{(32) r}{T}-K_{4} \stackrel{(21) r}{T}, \\
& 2 \stackrel{(62) r}{T}=-2 \stackrel{(53) r}{T}-\stackrel{44 r}{T}-2 K_{2} \stackrel{(42) r}{T}-K_{2}{ }^{33 r}-K_{4} \stackrel{22 r}{T}+K_{6} \stackrel{11 r}{T} \text {, } \\
& \stackrel{(63) r}{T}=-\stackrel{(54) r}{T}-K_{2}{ }^{(43) r} T+K_{6} \stackrel{(21) r}{T}, \\
& 2 \stackrel{(64) r}{T}=-\frac{55 r}{T}-K_{2} \stackrel{44 r}{T}+K_{4} \stackrel{33 r}{T}+2 K_{6} \stackrel{(31) r}{T}+K_{6} \stackrel{22 r}{T}, \\
& \stackrel{(65) r}{T}=K_{4} \stackrel{(43) r}{T}+K_{6} \stackrel{(41) r}{T}+K_{6} \stackrel{(32) r}{T}, \\
& \stackrel{66 r}{T}=K_{2} \stackrel{55 r}{T}+2 K_{4} \stackrel{(53) r}{T}+2 K_{6}{ }^{(51) r} T+K_{4} \stackrel{44 r}{T}+2 K_{6} \stackrel{(42) r}{T}+K_{6} \stackrel{33 r}{T} \text {. }
\end{aligned}
$$

For the second class with the second category,

$$
\begin{gathered}
\stackrel{(41) r}{T}=-\stackrel{(32) r}{T}-K_{2} \stackrel{(21) r}{T}, \\
2 \stackrel{(42) r}{T}=-\stackrel{33 r}{T}-K_{2} \stackrel{22 r}{T}+K_{4} \stackrel{11 r}{T}, \\
\stackrel{(43) r}{T}=K_{4} \stackrel{(21) r}{T}, \\
{ }^{44 r}=K_{2} \stackrel{33 r}{T}+2 K_{4} \stackrel{(31) r}{T}+K_{4} \stackrel{22 r}{T} .
\end{gathered}
$$

For the second class with the first category,

$$
\stackrel{(21) r}{T}=0, \quad \stackrel{22 r}{T}=K_{2} \stackrel{11 r}{T} .
$$

Proof. We first note that the terms in the right-hand side of [1, (3.11)] vanish identically when $x=y$. Therefore, whenever we use [1, (3.11)], it suffices to consider the terms corresponding to the cases $x \neq y$ only. The proof of the above relations follow from [1, (3.11)], using (15) for the proof of (25), (16) for the proof of (26), and (17) for the proof of (27), respectively. For example, relation (25b) may be proved as follows:

$$
\begin{aligned}
& 2 \stackrel{(62) r}{T}^{2}=2 \stackrel{(62) r}{T} \omega \mu \nu
\end{aligned}
$$

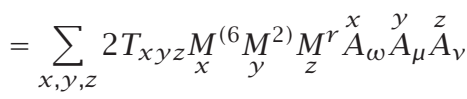




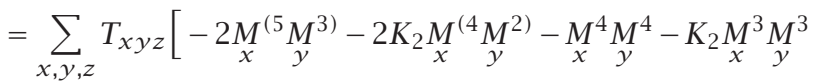

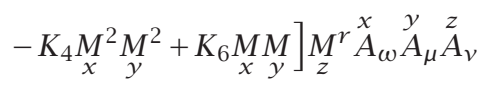

$$
\begin{aligned}
& =-2 \stackrel{(53) r}{T}_{\omega \mu \nu}-2 K_{2} \stackrel{(42) r}{T}_{\omega \mu \nu}-\stackrel{4}{T}_{\omega \mu \nu}-K_{2}{ }^{33 r} \omega \mu \nu \\
& -K_{4} \stackrel{22 r}{T} \omega \mu \nu+K_{6} \stackrel{11 r}{T} \omega \mu \nu \\
& =-2 \stackrel{(53) r}{T}-2 K_{2} \stackrel{(42) r}{T}-\stackrel{44 r}{T}-K_{2} \stackrel{33 r}{T}-K_{4} \stackrel{22 r}{T}+K_{6} \stackrel{11 r}{T} \text {. }
\end{aligned}
$$

ACKNOWLEDGMENT. This work was partially supported by the Basic Science Research Institute Program, Ministry of Education, Republic of Korea, 1997, BSRI-97-1442.

\section{REFERENCES}

[1] M. A. Kim, K. S. So, C. H. Cho, and K. T. Chung, Seven-dimensional considerations of Einstein's connection I. The recurrence relations of the first kind in $n-g-U F T$, Int. J. Math. Math. Sci. 2003 (2003), no. 12, 777-787.

Mi Ae Kim: Department of Mathematics, Yonsei University, Seoul 120-749, Korea

Keum Sook So: Department of Mathematics, Hallym University, Chunchon 200-702, Korea

E-mail address: ksso@sun.ha17ym.ac.kr

Chung Hyun Cho: Department of Mathematics, Inha University, Inchon 402-751, Korea

E-mail address: chcho@math.inha.ac.kr

Kyung Tae Chung: Department of Mathematics, Yonsei University, Seoul 120-749, Korea

E-mail address: ktchung@yonsei .ac.kr 


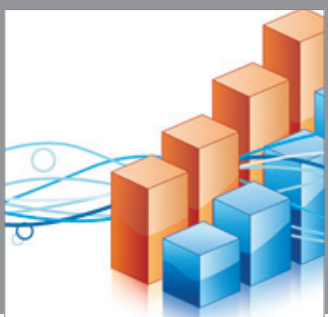

Advances in

Operations Research

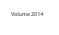

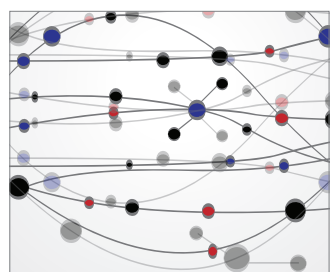

\section{The Scientific} World Journal
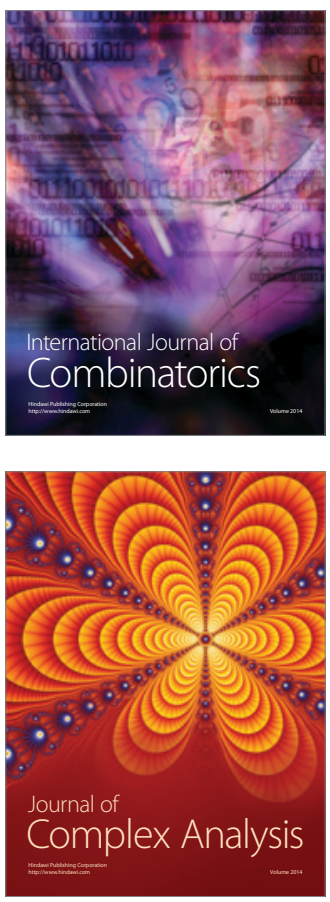

International Journal of

Mathematics and

Mathematical

Sciences
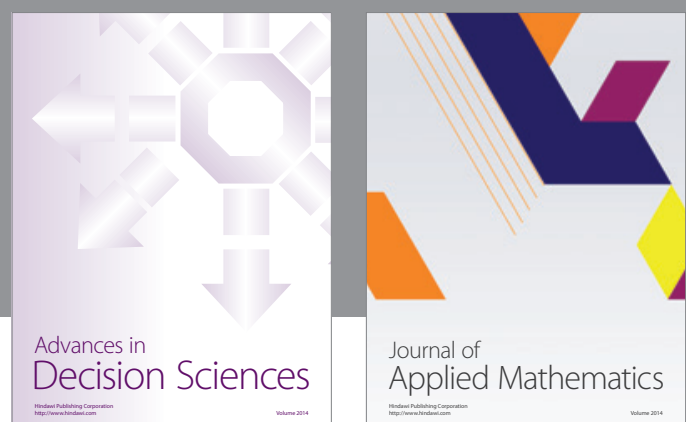

Journal of

Applied Mathematics
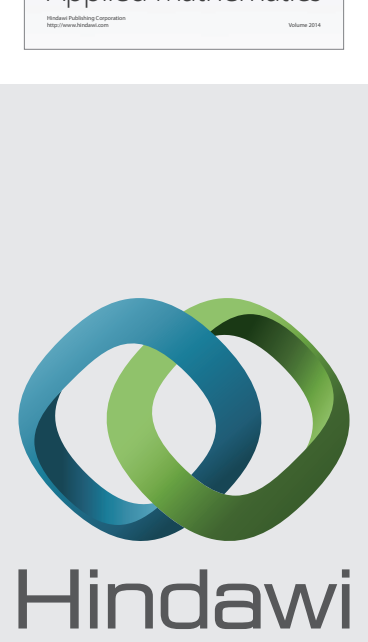

Submit your manuscripts at http://www.hindawi.com
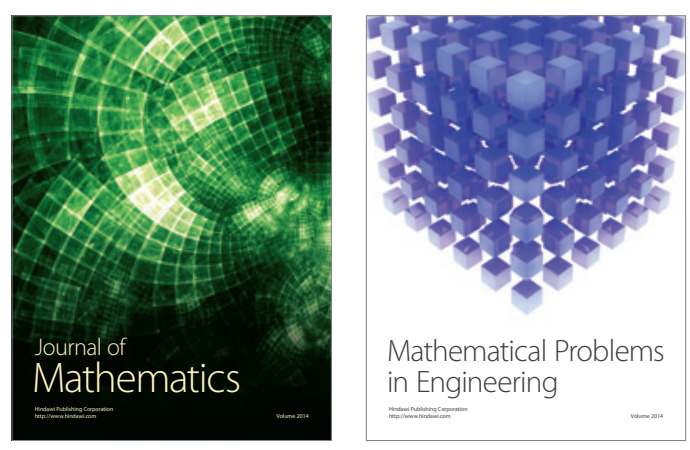

Mathematical Problems in Engineering
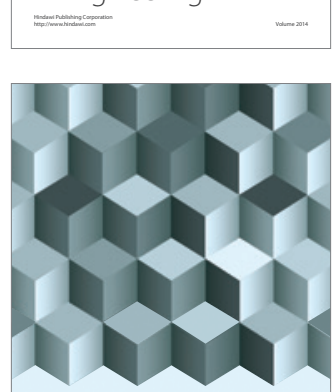

Journal of

Function Spaces
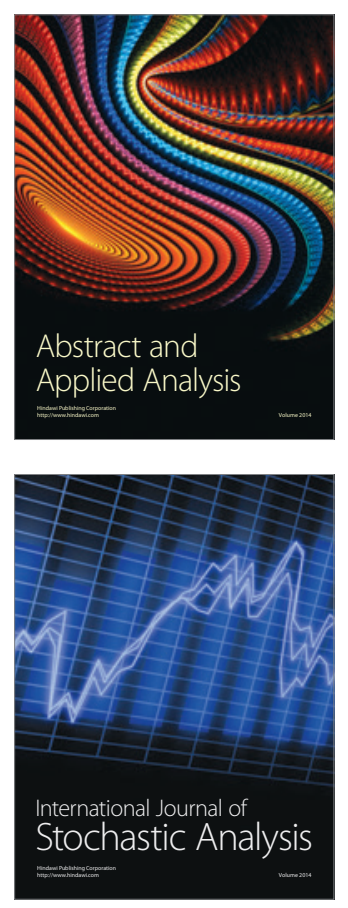

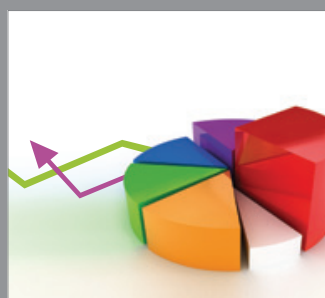

ournal of

Probability and Statistics

Promensencen
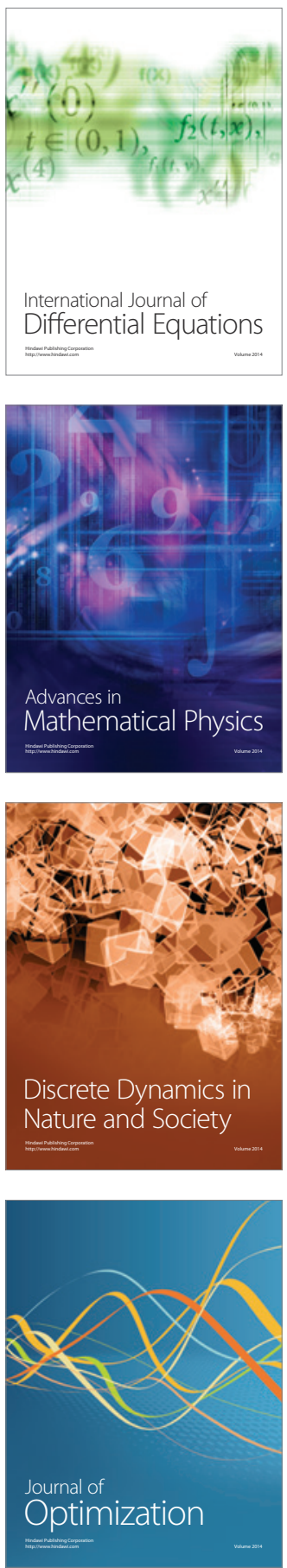